\title{
Design of a Sieve Plate Coin Screening Machine Zhao Yan ${ }^{1, a}$, Zhou Yan ${ }^{1, b}$, Hong Can ${ }^{1}$ \\ ${ }^{1}$ The Institute of Mechatronical Engineering and Automation ,Wuchang Shouyi University,Wuhan,430064, China \\ aemail: zhaoyanyz@qq.com, bemail:348399726@qq.com
}

Keywords: Coin Screening; Sieve Plate; False Identification

\begin{abstract}
At present counting and sorting of the coin depends on artificial means commonly, so the sorting and counting process is low efficiency and higher labor intensity, with a high cost of production. To solve this problem, a sieve plate coin screening machine was developed. This machine is mainly composed of the paper money - coin separation device, coin screening device, one-yuan coin counterfeit discrimination device, counting device, etc.It achieves a better effect of the paper currency and coin separation and the coin sorting of different face value, through the multiple sets of slider-crank mechanism and specially designed sieve plate.In its control system, the LDC1000 sensor can judge the true or false coins by extracting eigenvalues from the coins. One-yuan coin counterfeit discrimination device uses the steering gear to control the positive and reverse rotation of the sorting wheel to realize the separation of the one-yuan true and false coins. The weight of the machine is $40 \mathrm{~kg}$, and the sorting efficiency can be up to $600 \mathrm{pieces} / \mathrm{min}$. The machine has a good practical prospect in banks, bus stations and so on .
\end{abstract}

\section{Introduction}

Coin is one of the most commonly used currencies in the world, and it is widely used in commerce, transportation, communication, entertainment and a variety of vending and payment systems. Owing to the emergence of various new automatic payment systems, the field of coin payment is further expanded, which also makes the use of coins increase with each passing day [1]. At present, the classification of coins mainly relies on labor, the labor cost is high and efficiency is low. To solve this problem, this paper designs a kind of sieve-plate coin screening machine, and conducts the clearing test. The machine can finish coins clearing, counting and false identification at one time to reduce manual input, greatly improving the efficiency of coins clearing and satisfying the needs of clearing coins in banks, bus stations and so on.

\section{Overall structure and working principle}

The design of sieve-plate coin counting machine adopts inclined vibrating sieve plate design, and uses 12V FB775 DC gear motor to drive the sieve plate to move. The working device comprises paper money - coin separation device, coin screening device, one-yuan coin counterfeit discrimination device, counting device and rack. The overall structural design is shown in Figure 1 and Figure 2. 


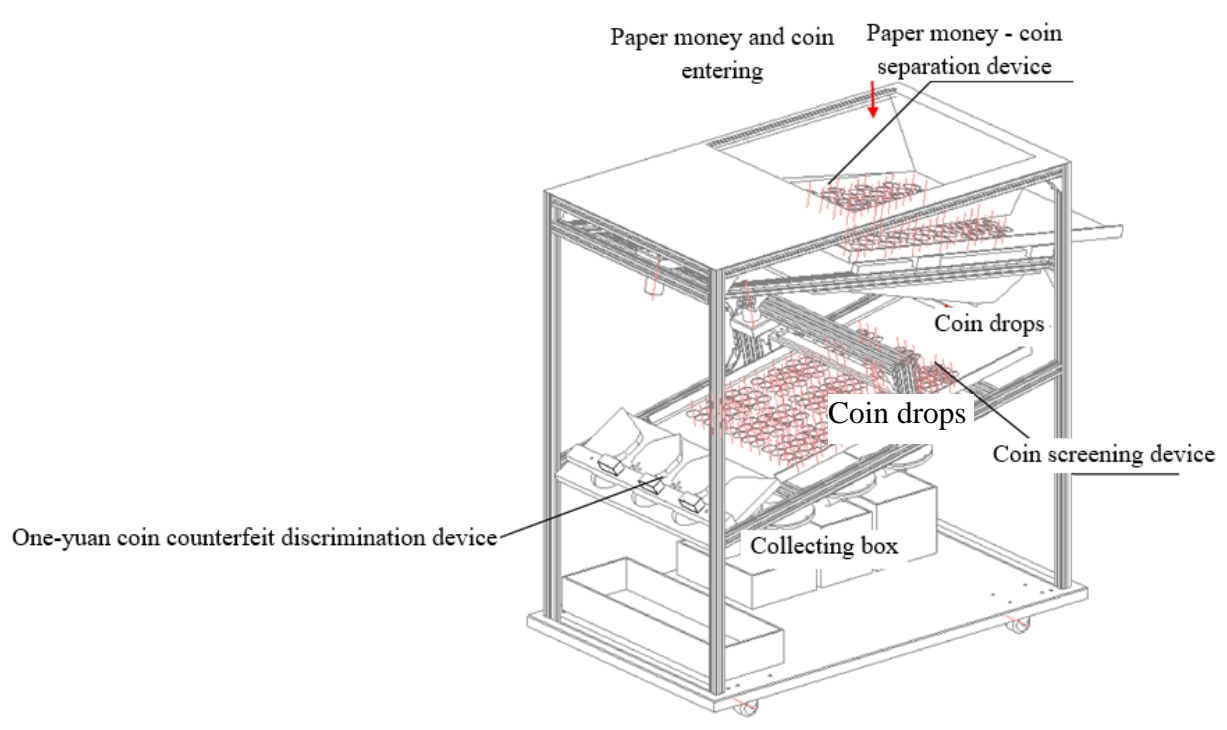

Figure.1. General assembly drawing (1)

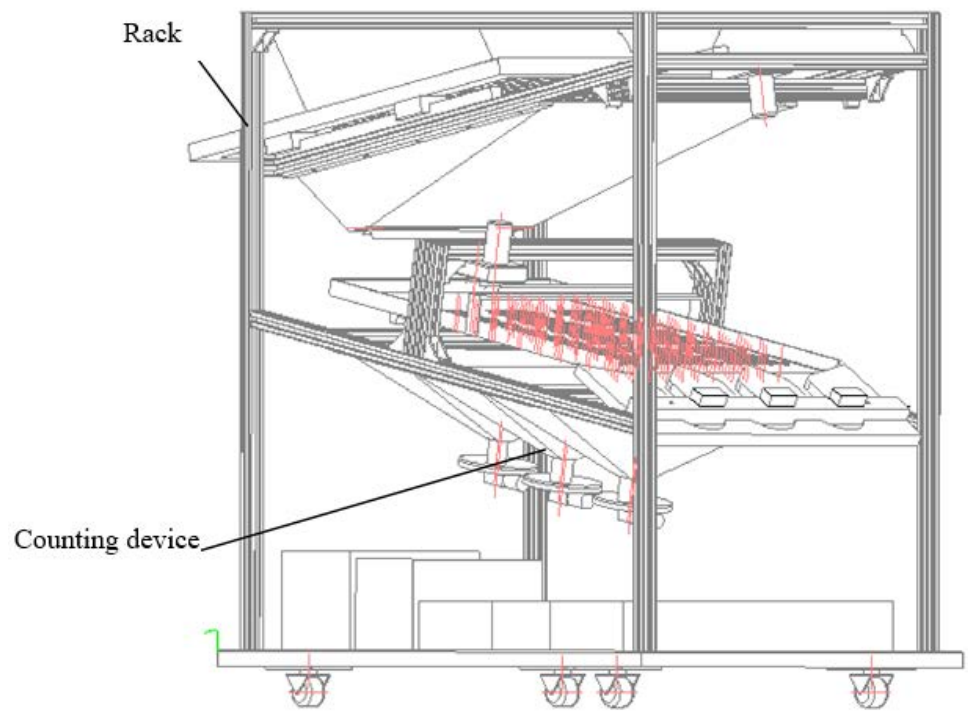

Figure.2.General assemblydrawing (2)

Paper money and coins enter into the counting machine from the top of the paper money-coin separation device, and the motor in the paper money- coin separation device drives crank slider mechanism to move and thus drives the stainless steel sieve of $10^{\circ}$ with the horizontal tilt angle to slide left and right to complete the screening and separation of paper money and coins. The paper money retains in the sieve plate, and the coins fall down, as shown in Figure 1.

The coin descends from the hopper into the coin screening device. The screening device of coins adopts the centric slider-crank mechanism, uses the sieve plate as the slide block, makes the straight reciprocating movement, uses the different size apertures and carries out the screening of the coin. Except one-yuan coin, the other face value coins enter the counting device, and drop to the collecting box, as shown in Figure 2. In order to make the counting more stable, the roulette and chute mechanism are used so that the coins are individually scanned by the infrared tube for counting, and the touch screen displays data.

The one-yuan coin slides from the coin screening device to the one-yuan coin counterfeit discrimination device, as shown in Figure 1. One-yuan coin counterfeit discrimination device partly uses slide and sorting wheel mechanism, the coins enter into the sorting wheel through the slide, and the sorting wheel rotates positively and reversely in order to achieve separation of one-yuan true and false currency. One-yuan coin is counted by feedback data of LDC1000 sensor, and then the quantity is displayed by the touch screen. One-yuan coins pass the LDC1000 sensor. By the use of eddy current effect, the eigenvalue sampling of the coin is carried out so as to determine the true and false coins. The stm32 single-chip microcomputer controls MG996R steering gear to achieve 
the positive and reverse rotation of the specified angle $\left(60^{\circ}\right)$, the true and false coins fall from the different holes, and collection can be done.

The final design size parameters of the machine are $770 \mathrm{~mm} \times 420 \mathrm{~mm} \times 700 \mathrm{~mm}$, the weight is $40 \mathrm{~kg}$, and the sorting efficiency can be up to 600 pieces $/ \mathrm{min}$.

\section{Design of key working mechanisms}

\section{Design of coin screening device}

The device uses the centric slider-crank mechanism. After repeated tests, the tilt angle of the base is $10^{\circ}$ so as to ensure that the coins slide down at an appropriate speed along the sieve plate to avoid leaking the coin or stacking coin. Through the different sieve aperture sizes (Figure 3), the holes are staggered to facilitate the coins into the funnel, and then sieve plate vibrates to achieve the screening of coins.

The specific structure of the coin screening device is shown in Figure 3 below:

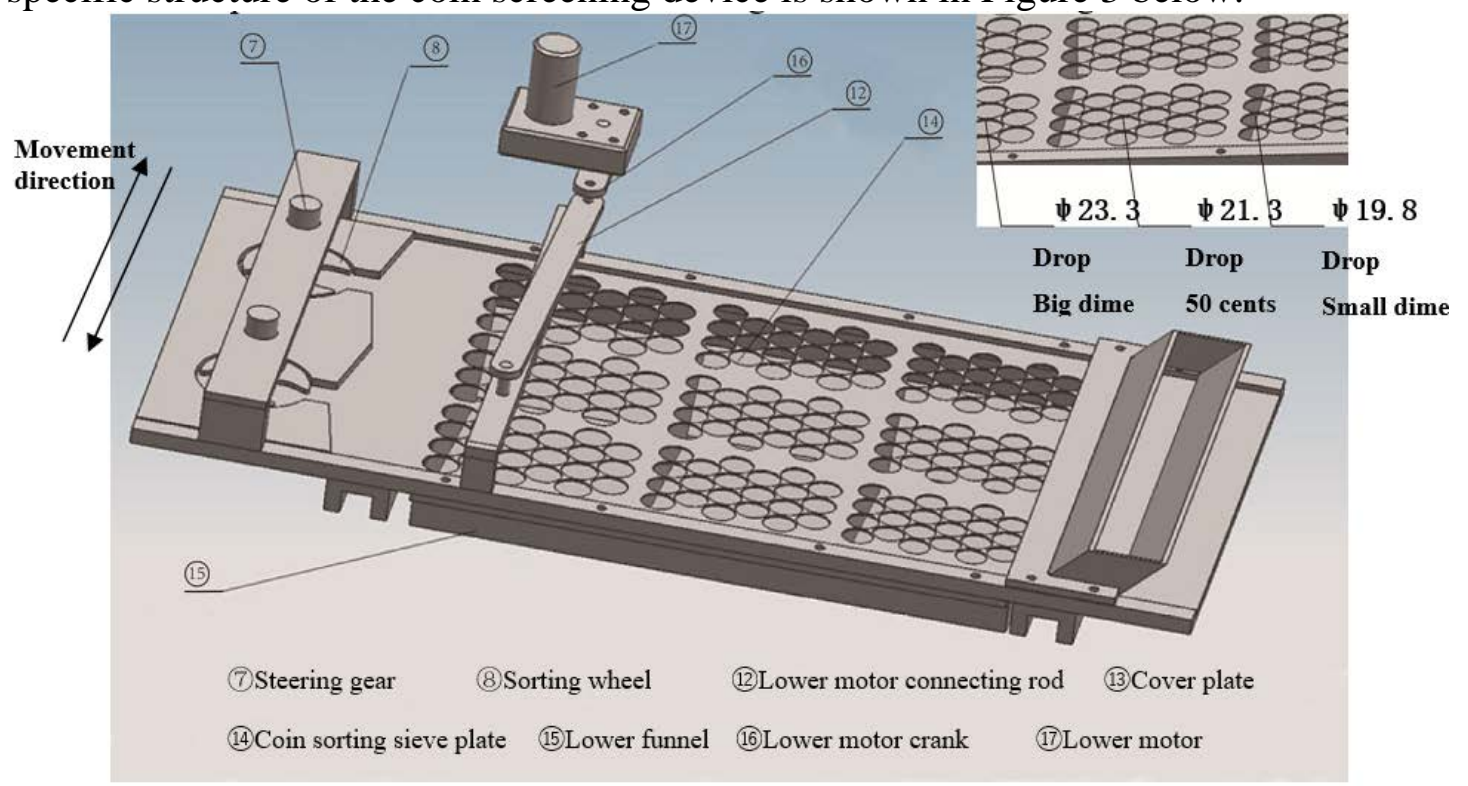

Figure.3.Coin screening device

Coins pass through the upper funnel

(B) Ththootgh silmediffatent sieve aperture sizes and the appropriate vibration, a dime and fifty-cent coins fall into the lower funnel (15). By the use of the infrared light tube, count the coins and display on the display screen. One-yuan coins continue to move towards the rear end of the sieve plate and enter into the next unit.

One-yuan coin counterfeit discrimination device

The device is a simple one-level mechanism; the steering gear directly drives the sorting wheel to achieve clearing and sorting of true and false coins. The specific structure is shown in Figure 4.

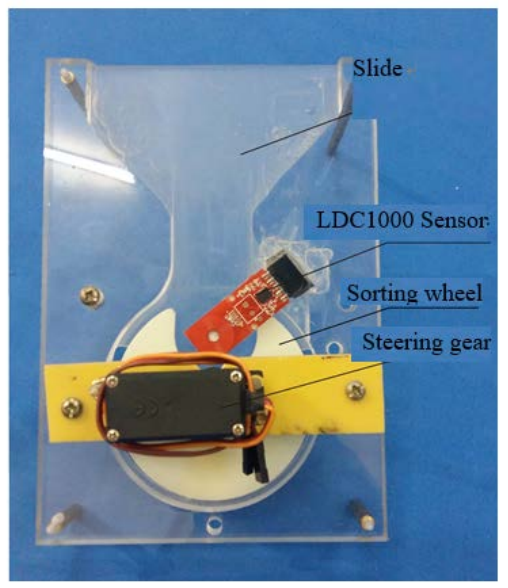

Figure.4.One-yuan coin anti-counterfeiting device (Model) 
When one-yuan coin leaves the sieve plate, passes the slide and enters the sorting wheel, the LDC1000 sensor collects the resistance and inductance of the coin for eigenvalue sampling of the coin (Figure 5), the data processing has been greatly optimized, and the coin inductance is also introduced into the program for discrimination (Figure 6). Combine with the early distance eigenvalue to determine the true and false of the coin. The stm32 single-chip microcomputer controls MG996R steering gear to achieve the positive and reverse rotation of the specified angle $\left(60^{\circ}\right)$. At the same time, the counterfeit money warning red light in the touch screen lights up, the alarm sends out an alarm, the real money and counterfeit money fall from different holes, the real money falls into the large collecting box, and the counterfeit money into the special collecting box. Counting is achieved by the feedback data of the LDC1000 sensor, which is then displayed through the LCD display.

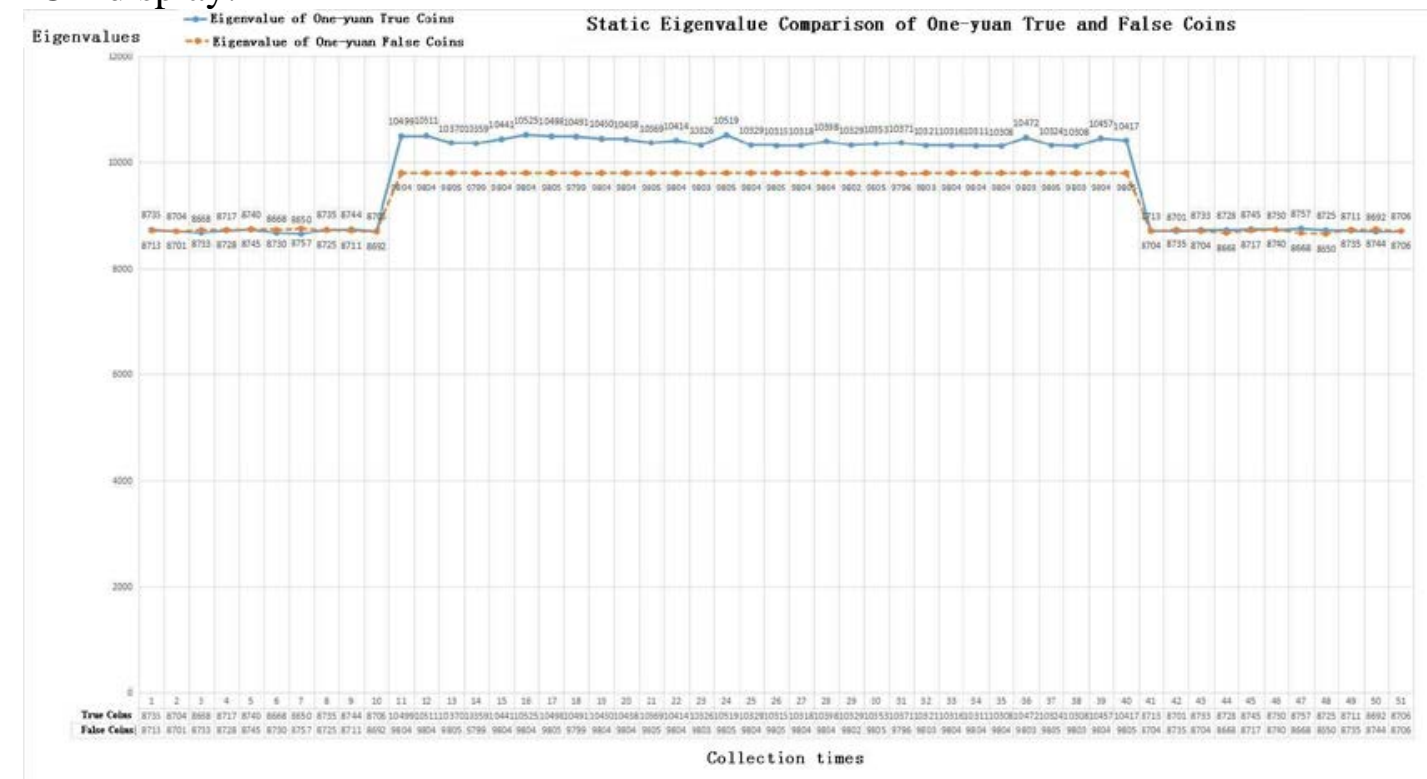

Figure.5. Static eigenvalue comparison of one-yuan true and false coins

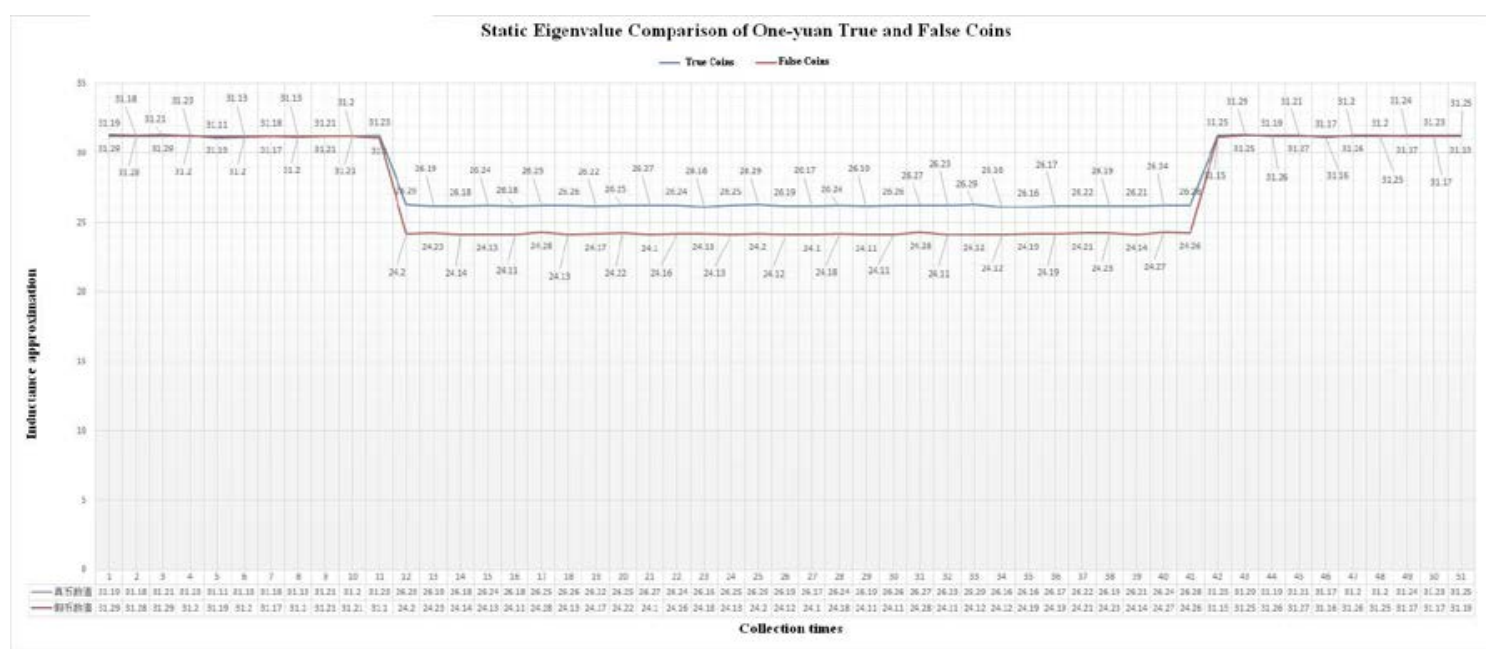

Figure.6.Inductance approximation comparison of true and false coins under $27^{\circ} \mathrm{C}$ room

temperature

Design of the count part

The counting of a dime and fifty cents is done by movable plate, chute structure and several phototubes. By using the movable plate and the chute structure, the coins are passed through the phototubes tube one by one, making the counting more accurate and reliable, as shown in Figure 7. One-yuan coin is counted by feedback data of LDC1000 sensor, and then the quantity is displayed by the display screen. 


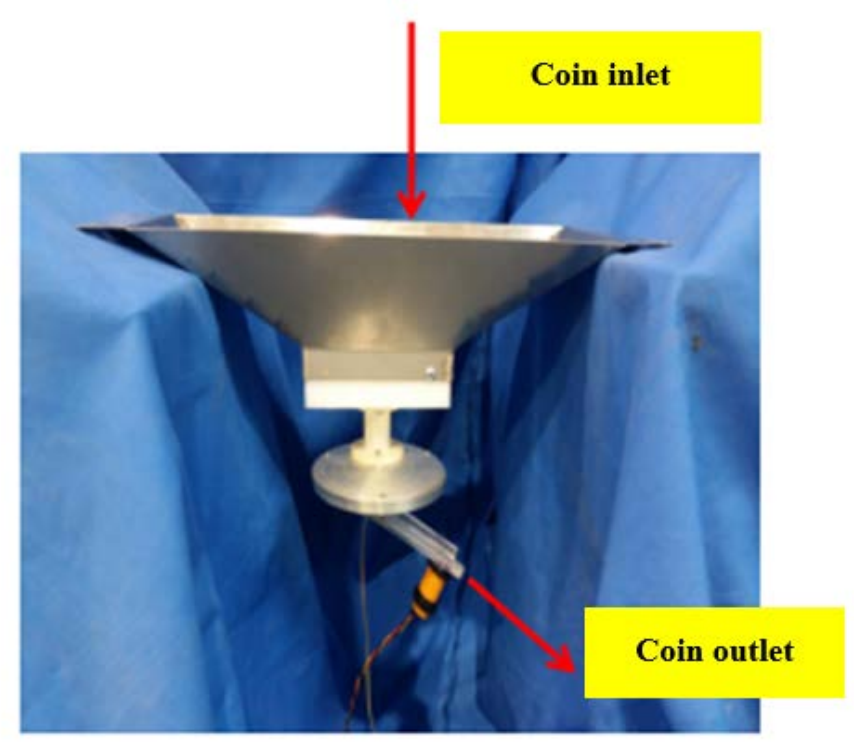

Figure.7.Counting device

\section{Design of the control part}

The counting machine adopts the stm32f103zet6 single-chip microcomputer to control the motor part. The BTS7970 drive board controls the motor to separate the paper money and the coin, and then the L298N driver board controls the steering gear to sort the true and false coins. By using the minimum system of 51 single-chip microcomputer, LCD screen and a number of phototubes for coin counting, the specific program is shown below.

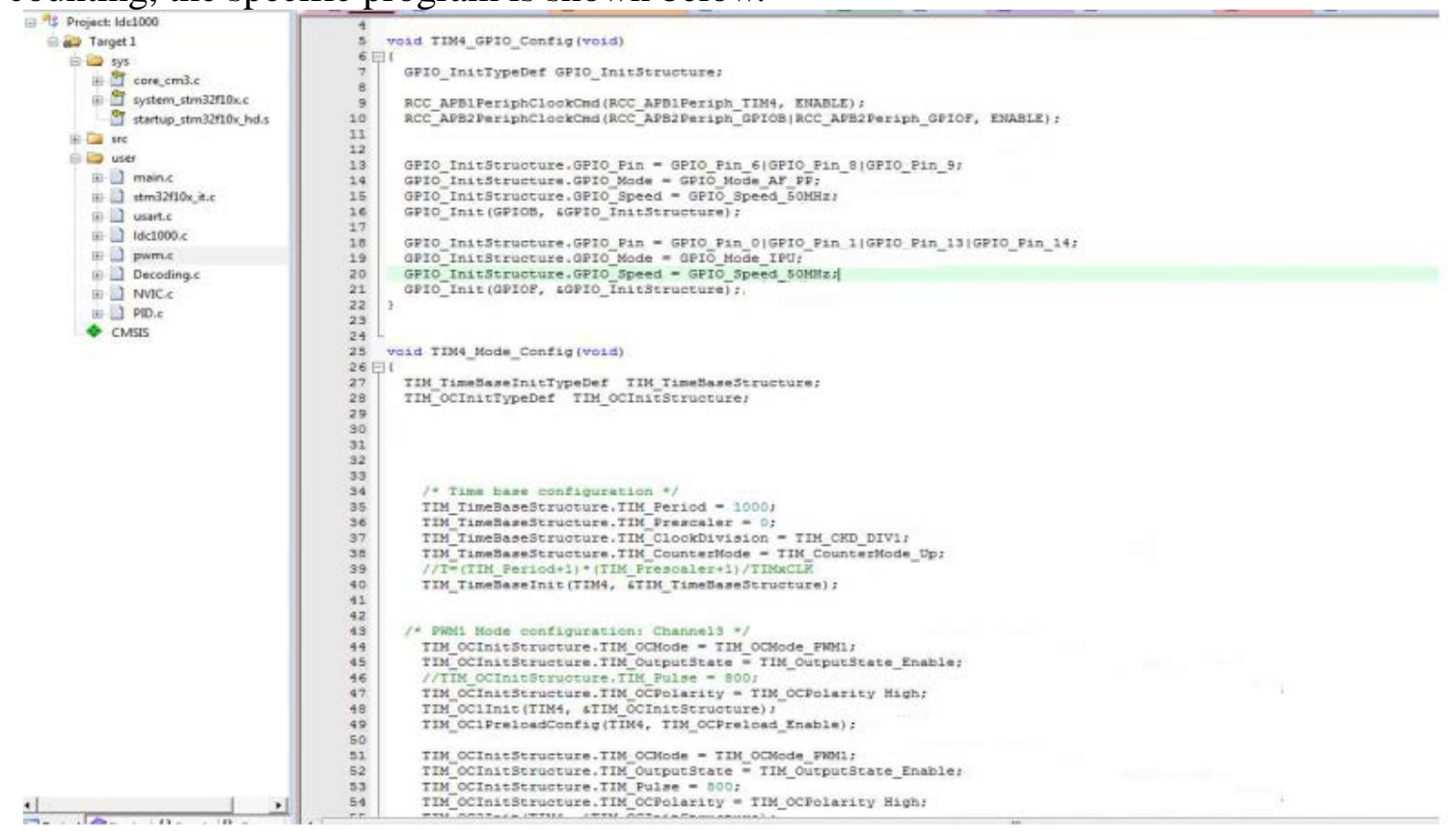

Figure.8.Part of the control program

\section{Conclusion}

The coin counting machine studied in this paper is composed of paper money-coin sorting device, coin screening device, one-yuan coin counterfeit discrimination device, counting device and rack. Among them, the coin screening section has a sieve plate with $10^{\circ}$ inclination angle. By use of different apertures, coin sieving is conducted. The clearing and sorting are accurate with high efficiency, up to 600 pieces/min. One-yuan coin counterfeit discrimination device uses the steering 
gear to control the positive and reverse rotation of the sorting wheel to realize the separation of the one-yuan true and false coins. The data processing is greatly optimized, and the coin inductance is also introduced into the program for judgment. Distance eigenvalues are combined so that the entire anti-counterfeiting device is more stable and reliable. Coin counting adopts a movable plate and chute structure so that coins slide one by one. Combined with photoelectric sensors, counting is more accurate and reliable. The size of the coin counting machine is $770 \mathrm{~mm} \times 420 \mathrm{~mm} \times 700 \mathrm{~mm}$ and the weight is $40 \mathrm{~kg}$. The coin counting machine has medium size, light weight, high efficiency and low cost and can complete discrimination of counterfeit coins, which is suitable for banks, bus stations and other occasions that need counting a large number of coins to solve the problem of artificial counting and greatly reduce labor costs. It has a promising application prospect.

\section{References}

[1] Guo Xingi,Shang Yunan. Modeling Design Research of ST800A Coin Counting Machine. Packing Engineering. 2007, (08): 164-169.

[2] Li Qun, Lu Yuxia. Thoughts on Coin Counting Processing Work [J]. Jilin Finance Research, 2012, (07): 68-69.

[3] Huang Min, Zhu Xiaolin, Shen Ying, et al. Development of Coin Automatic Classifier [J]. Development and Innovation of Electromechanical Products, 2013, 26 (06): 43-45.

[4] Sun Huan, Chen Zuomo. Mechanical principle. The 7th edition (Beijing): Higher Education Press, 2013.

[5] Ying Yuming. Design of Automatic Counting System of Workpiece Based on Single Chip Microcomputer Control [J]. Mechanical \& Electrical Engineering Technology, 2011, (07): 47-49.

[6] Si Yunyun and Jing Qinqin. Design and Fabrication of Infrared Photoelectric Counter [J]. Electronic Manufacturing, 2014, (10): 10-11. 